\title{
SINAIS E SINTOMAS OSTEOMUSCULARES RELACIONADAS AO TRABALHO EM PROFISSIONAIS DE ENFERMAGEM
}

\author{
SIGNS AND SYMPTOMS MUSCULOSKELETAL RELATED \\ TO WORK IN NURSING PROFESSIONALS
}

\section{Camila Gonçalves Monteiro Anunciação', Luciana Aparecida Sales', Mileid Correa Andrade', Cristiane Aparecida Silveira², Sônia Maria Alves Paiva²}

\begin{abstract}
RESUMO
Objetivo: identificar e caracterizar a percepção de sinais e sintomas osteomusculares relacionados ao trabalho da enfermagem em um hospital. Metodologia: estudo descritivo, quantitativo e epidemiológico, realizado com 25 profissionais de enfermagem de uma unidade de internação clínica. Foram entrevistados utilizando um instrumento semiestruturado adaptado, contendo informações sobre percepção de dor dados pessoais e demográficos dos participantes. Resultado: $20(80 \%)$ eram mulheres, $23(92 \%)$ trabalhavam 48 horas semanais (12/36 horas), 10 (40\%) tinham idade entre 2837 anos, 11 (44\%) tinham de um a três anos de tempo de serviço, 19 apresentavam sintoma de dor, mas somente oito (32\%) procuraram tratamento. Todos realizaram tratamento farmacológico, $6(24 \%)$ realizaram acupuntura, 1 (4\%) realizou fisioterapia convencional e 1 (4\%) hidroterapia. Conclusão: Os participantes identificam os sinais e sintomas, entretanto não buscam tratamento. Seria recomendado reorganizar os espaços de trabalho, fomentar a implementação de programas de reeducação postural e de programas com enfoques em ergonomia.
\end{abstract}

Descritores: Sinais e Sintomas; LER/DORT; Enfermagem.

\begin{abstract}
Objective: To describe the perception of musculoskeletal signs and symptoms related to nursing work at a hospital. Method: A descriptive, quantitative and epidemiological study conducted with 25 nursing professionals of a clinical inpatient unit. The interviews were conducted using a semi-structured instrument adapted. Results: 20 (80\%) were women, 23 (92\%) worked 48 hours a week (12/36hours),, $10(40 \%)$ were between $28-37$ years old, $11(44 \%)$ ranged from one to three years of service time, 19 had symptoms of pain, but only eight (32\%) sought treatment. All of the participants underwent drug treatment, $6(24 \%)$ underwent acupuncture treatment, $1(4 \%)$ engaged conventional therapy of physiotherapy and $1(4 \%)$ underwent a hydrotherapy treatment. Conclusion: The participants identified signs and symptoms, however they did not seek treatment. Would be recommended to organize workspaces, further the implementation of postural rehabilitation programs with ergonomic approach.
\end{abstract}

${ }^{1}$ Graduada em Enfermagem pela Pontifícia Universidade Católica de Minas Gerais (PUC Minas) Brasil, Belo Horizonte, MG, Brasil.

${ }^{2}$ Doutora em Enfermagem pela Escola de Enfermagem de Ribeirão Preto da USP (EERPUSP), Ribeirão Preto, SP, Brasil. 


\section{Introdução}

A sociedade é marcada por intensas e rápidas transformações, que afetam a vida humana tanto em seus aspectos coletivos quanto individuais. $O$ trabalho pode ser definido como o conjunto de elementos ou circunstâncias que estejam relacionados a material, psíquico, biológico e social. Sendo assim, o processo de trabalho é diferenciado e único para cada ser, o que determina de que forma se desenvolve a atividade laboral'1.

As relações entre 0 trabalho e 0 trabalhador são estabelecidas a partir de diferentes formas de organização e gestão, que podem levar ao adoecimento profissional'2. A gestão do trabalho pode influenciar na ausência do trabalho (absenteísmo), no afastamento por auxílio-doença e no remanejamento de recursos humanos, ocasionando grande problema de pessoal para as organizações e seus gestores. Pode gerar também o aumento nos custos em saúde do trabalhador e ainda afetar a qualidade dos serviços prestados ${ }^{3}$.

Trabalhadores da área da saúde, como por exemplo, enfermeiros e técnicos de enfermagem, têm alto risco para doenças relacionadas ao trabalho, devido a constante exposição aos riscos ocupacionais, esforço físico, posições inadequadas, movimentos repetitivos e levantamento de peso $^{4}$, especialmente em instituições hospitalares em que a demanda de esforço físico é maior em razão de sua complexidade e estresse físico ${ }^{5}$, gerando danos diversos, especialmente lesões osteomusculares ${ }^{6}$, cuja principal característica é a dor crônica.

As lesões mais comuns em ambientes de trabalho são a Lesão por esforço repetitivo (LER) e os Distúrbios Osteomusculares Relacionados ao Trabalho (DORT). A LER se caracteriza por dor localizada em músculos, tendões, nervos e vasos dos membros superiores e inferiores ocasionando desconforto, parestesia, sensação e diminuição real de força, aumento de massa corporal, fadiga, edema e enrijecimento articular, podendo ser causada por esforços desvinculados ao trabalho ${ }^{7}$. Já os Distúrbios Osteomusculares Relacionados ao Trabalho (DORT) apresentam os mesmos sinais e sintomas da LER, porém possui relação direta com as tarefas desenvolvidas no trabalho, o ambiente físico do qual o trabalhador está inserido e a organização do trabalho ${ }^{7}$. No Brasil, foi adotada formalmente a sigla DORT, seguindo a tendência internacional de enfatizar a relação com o trabalho². Porém, como a sigla LER já era amplamente conhecida, optou-se por mantê-la, consolidando-se assim a junção LER/DORT ${ }^{8}$.

Apesar dos termos derivarem basicamente da medicina, sua causas são multifatoriais: fatores biomecânicos (carga de trabalho, esforço repetitivo, posturas inadequadas e vibrações corporais), psicossocial e organizacional e multidimensional (dimensão individual, grupal e social) ${ }^{8}$ e abrangem um grupo heterogêneo de distúrbios do sistema musculoesquelético incluindo queixas de dor, formigamento, dormência, peso e fadiga, afetando principalmente músculos, tendões, nervos e ligamentos ${ }^{7}$.

A LER/DORT é um grave problema de saúde do trabalhador, atinge distintas classes profissionais e pode gerar diferentes graus de incapacidade funcional ${ }^{9}$. Devido alta prevalência de doenças osteomusculares, o Ministério da Saúde tornou de notificação compulsória os agravos relacionados ao trabalho, entre os quais estão a LER/DORT ${ }^{10}$. A notificação é fundamental para o planejamento de ações de prevenção e promoção da saúde com base nos números reais de trabalhadores afetados. Aliada a isso é fundamental a disseminação de conhecimento e criação de uma cultura prevencionista, visando auxiliar os profissionais de saúde na prevenção, tratamento e reabilitação dos LER/DORT ${ }^{1}$.

Dentre as profissões da área da saúde, a enfermagem, tem sido especialmente afetada pelo distúrbio musculoesquelético. Pesquisas realizadas em vários países exibem prevalências superiores a $80 \%$ de ocorrência de distúrbios musculoesqueléticos em trabalhadores de enfermagem no Japão ${ }^{11}$, na China ${ }^{12}$ e em Portugal ${ }^{13}$. No Brasil, estudos mostram prevalências de até $93 \%$ desses distúrbios, contribuindo significativamente para a incapacidade e 0 afastamento do trabalho ${ }^{14}$.

Apesar das altas prevalências das LER/DORT nos ambientes hospitalares, os trabalhadores têm dificuldades em identificá-las seja pela demora do diagnóstico, seja pela neutralização da dor.

Diante do exposto, o objetivo do presente trabalho foi identificar e caracterizar a percepção de sinais e sintomas osteomusculares relacionados ao trabalho hospitalar da enfermagem. 


\section{Metodologia}

Trata-se de uma pesquisa exploratória de abordagem quantitativa de caráter descritivo realizada no setor de internação cirúrgica de uma instituição de saúde. A escolha deste setor foi devido aos tipos de cuidados prestados pela enfermagem que predispõem o aparecimento de inúmeras lesões e distúrbios relacionados ao trabalho. Neste setor os enfermeiros dedicam grande parte do tempo às atividades administrativas, mas também realizam atividades assistenciais como banhos, curativos, transferências do paciente das macas para as camas dentre outras. Os técnicos e auxiliares de enfermagem realizam atividades assistências como as citadas anteriormente que demandam esforço físico e carregamento de peso.

O setor era composto por 28 funcionários. Entretanto dois estavam de férias e um recusou-se a participar, resultando em 25 participantes (três exercem na clínica cirúrgica a função de enfermeiro; 19 exercem a função de técnico em enfermagem e três exercem a função de auxiliar de enfermagem).

Para a coleta de dados utilizou-se um instrumento tipo questionário semiestruturado, adaptado ${ }^{15}$. 0 questionário contém questões sobre aspectos sociodemográficos, história ocupacional no emprego atual e vida laboral pregressa e questões sobre dores relacionadas ao trabalho em profissionais de enfermagem como localização, tipos, dentre outros. Não foram avaliadas questões psicológicas como satisfação com o trabalho e/ou motivação.

As entrevistas foram agendadas com os participantes da pesquisa. Esse agendamento foi pré-acordado com a gerência institucional. Antes da entrega dos instrumentos, os participantes foram orientados quanto aos objetivos do trabalho, bem como a importância da pesquisa. Depois de sanadas todas as dúvidas, a pesquisadora entregou o Termo de Consentimento Livre Esclarecido (TCLE) aos participantes para procederem à leitura, consentimento e assinatura. Em seguida procedeu-se a aplicação dos instrumentos de coleta.

Foram incluídos os técnicos de enfermagem e enfermeiros maiores de 18 anos, que consentiram em participar da pesquisa. Foram excluídos todos aqueles que não consentirem em participar da pesquisa ou que estavam afastados do trabalho (licença-maternidade, licença-saúde, férias, entre outros). A análise dos dados foi realizada a partir da estatística descritiva.

Para a realização deste estudo, respeitou-se as diretrizes da Resolução n. 466/12 do Conselho Nacional de Saúde ${ }^{16}$. $O$ estudo foi aprovado pela Instituição de Saúde e pelo Comitê de Ética em Pesquisa da Pontifícia Universidade Católica de Minas Gerais (CAAE 0069.0.213.000).

\section{Resultados}

Os dados biopsicossociais relativos aos 25 profissionais estudados estão apresentados na Tabela 1.

Tabela 1 - Distribuição dos trabalhadores da clínica cirúrgica de um hospital segundo seus dados biopsicossociais ( $n=25)$.

\begin{tabular}{c|c|c|c}
\hline \multirow{2}{*}{ Variáveis } & & \multicolumn{2}{|c}{ TOTAL } \\
\cline { 2 - 4 } & & 8 & $\%$ \\
\hline \multirow{4}{*}{ Faixa etária } & $18-27$ anos & 10 & 32 \\
\cline { 2 - 4 } & $28-37$ anos & 5 & 40 \\
\cline { 2 - 4 } & $38-47$ anos & 2 & 20 \\
\cline { 2 - 4 } & $48-57$ anos & 25 & 8 \\
\cline { 2 - 4 } & Total & & 100 \\
\hline \multirow{4}{*}{ Sexo } & Masculino & 5 & 20 \\
\cline { 2 - 4 } & Feminino & 20 & 80 \\
\cline { 2 - 4 } & Total & 25 & 100 \\
\hline
\end{tabular}

Continua... 


\begin{tabular}{|c|c|c|c|}
\hline \multirow{2}{*}{ Variáveis } & & \multicolumn{2}{|c|}{ TOTAL } \\
\hline & & $\mathbf{F}$ & $\%$ \\
\hline \multirow{5}{*}{ Anos de Trabalho } & 1-3 anos & 11 & 44 \\
\hline & 4-6 anos & 6 & 24 \\
\hline & 7-9 anos & 4 & 16 \\
\hline & Acima de 10 anos & 4 & 16 \\
\hline & Total & 25 & 100 \\
\hline \multirow{4}{*}{ Carga horária } & 48 horas $(12 / 36)$ & 23 & 92 \\
\hline & 36 horas & 1 & 4 \\
\hline & 48 horas & 1 & 4 \\
\hline & Total & 25 & 100 \\
\hline \multirow{3}{*}{$\begin{array}{l}\text { Possui outro } \\
\text { vínculo } \\
\text { empregatício }\end{array}$} & Sim & 7 & 28 \\
\hline & Não & 18 & 72 \\
\hline & Total & 25 & 100 \\
\hline
\end{tabular}

Legenda: $\mathrm{f}=$ frequência

Fonte: Dados da pesquisa

Constatou-se a predominância do sexo feminino com 20 (80\%) participantes e a idade predominante foi entre 28 e 47 anos onde se encontraram15 (60\%) participantes com idade média de 31 anos $( \pm 5,11)$.

Do total, 19 (76\%) informaram que já apresentaram um dos sinais/sintomas de LER/DORT, sendo eles o principal dor muscular. Outros sinais e sintomas foram dificuldade para movimentar os membros superiores e/ou inferiores, parestesias, fadiga muscular, alteração da temperatura e da sensibilidade local, redução na amplitude do movimento e/ou inflamação.

Tabela 2 - Distribuição dos profissionais de enfermagem segundo sintomas, dor e tratamento de LER/DORT e afastamento do trabalho $(n=25)$.

\begin{tabular}{c|c|c|c}
\hline \multirow{2}{*}{ Variáveis } & & \multicolumn{2}{|c}{ TOTAL } \\
\cline { 2 - 4 } & & $\mathbf{F}$ & $\%$ \\
\hline \multirow{3}{*}{$\begin{array}{c}\text { Sintomas de LER/ } \\
\text { DORT }\end{array}$} & Sim & 19 & 76,0 \\
\cline { 2 - 4 } & Não & 6 & 24,0 \\
\cline { 2 - 4 } & Total & 25 & 100,0 \\
\hline & \multicolumn{3}{|c}{} \\
\cline { 2 - 4 } & Braço & 12 & 63,2 \\
\cline { 2 - 4 } & Punho & 8 & 42,1 \\
\cline { 2 - 4 } $\begin{array}{c}\text { Localização dos } \\
\text { sinais e sintomas* }\end{array}$ & Pescoço & 8 & 42,1 \\
\cline { 2 - 4 } & Ombro & 7 & 36,8 \\
\cline { 2 - 4 } & Lombar & 6 & 31,6 \\
\cline { 2 - 4 } & $\begin{array}{c}\text { Outras } \\
\text { localizações }\end{array}$ & 7 & 36,8 \\
\hline
\end{tabular}

Continua. 


\begin{tabular}{|c|c|c|c|}
\hline \multirow{2}{*}{ Variáveis } & & \multicolumn{2}{|c|}{ TOTAL } \\
\hline & & $\mathbf{F}$ & $\%$ \\
\hline \multirow{6}{*}{$\begin{array}{c}\text { Tratamento além } \\
\text { do farmacológico } \\
\text { sob prescrição } \\
\text { médica }\end{array}$} & Acupuntura & 6 & 24,0 \\
\hline & $\begin{array}{l}\text { Fisioterapia } \\
\text { convencional }\end{array}$ & 1 & 4,0 \\
\hline & Hidroterapia & 1 & 4,0 \\
\hline & Sem tratamento & 11 & 44,0 \\
\hline & Não se aplica & 6 & 24,0 \\
\hline & Total & 25 & 100,0 \\
\hline \multirow{4}{*}{$\begin{array}{c}\text { Afastamento do } \\
\text { trabalho }\end{array}$} & Sim & 4 & 16,0 \\
\hline & Não & 15 & 60,0 \\
\hline & Não se aplica & 6 & 24,0 \\
\hline & Total & 25 & 100,0 \\
\hline
\end{tabular}

* Considerando-se os 19 sintomáticos

Quanto à localização das dores, 12 (48\%) identificaram dor nos braços e $8(32 \%)$ dor no punho.

Dos 19 (76\%) profissionais de enfermagem que sentiram sintomas do LER/DORT, oito (32\%) buscaram tratamento médico, sendo todos tratamento farmacológico sob prescrição. Além do tratamento farmacológico, seis (24\%) participantes realizaram acupuntura para melhoria do quadro álgico. Após o tratamento, quatro profissionais (16\%) informaram terem apresentado recidiva da algia, sendo classificada como dor frequente em dois profissionais.

Dos que apresentavam sinais e sintomas, quatro (16\%) dos profissionais necessitaram de afastamento (dois foram afastados entre um a sete dias e dois mais de 15 dias), sem recidivas. Considerando que $60 \%$ não foram afastados do trabalho, pode-se entender que os sinais e sintomas não foram limitante a ponto de levar o participante a afastamento ou ainda revelar um outro problema sério que é a dificuldade de diagnóstico e tratamento correto.

Das atividades realizadas, 21 profissionais (84\%) informaram que são situações provocadoras de dor logo após serem realizadas: banho de aspersão em paciente, retirada de paciente do leito (sem auxílio), sobrecarga de pacientes para cuidado e transporte de paciente em maca (sem auxílio). Entretanto dois relatarem que nunca sentiram dores crônicas. Ressalta-se que as informações foram relatadas pelos profissionais de enfermagem, sem observação dos pesquisadores nos locais de trabalho.

Em relação à avaliação do conhecimento sobre LER/DORT, os resultados denotam que sete (28\%) profissionais de enfermagem (3 auxiliares e 4 técnicos de enfermagem) referem não possuir conhecimento de como realizar a prevenção da LER/DORT.

Dentre os 25 profissionais de enfermagem, 16 (64\%) profissionais dos entrevistados informam que na instituição em que trabalham não recebem orientações sobre como proceder para evitar lesões que provoquem a LER/DORT e nove $(36 \%)$ informaram receber orientações. Os que informaram receber orientações, cinco relataram que a mesma ocorre mensalmente, sendo principalmente através de informativos institucionais. Essa diferença sobre capacitação pode ser resultado do fato de as mesmas serem ministradas de forma diferente entre os plantões e sem uma regularidade. Funcionários novos na instituição não participaram de treinamentos de anos anteriores.

\section{Discussão}

Idade e sexo são quesitos importantes no contexto laboral referente a LER/DORT. Considerando que a enfermagem é uma profissão predominantemente feminina e que vários estudos ${ }^{17,18}$ sugerem 0 aparecimento de problemas osteomusculares com o avançar da idade e como principal causa de absenteísmo, compreende-se a gravidade do problema para a enfermagem. 
Esta prevalência feminina tem diversas explicações e também consequências para a saúde da mulher, pois além do trabalho remunerado a mulher desempenha seu papel nas tarefas domésticas, cuidado com os filhos e ainda muitas fazem dupla jornada de trabalho, influenciando o processo de adoecimento das trabalhadoras de enfermagem, acrescida do fenômeno da feminilização de algumas doenças como LER/DORT ${ }^{19}$.

Este fenômeno se dá a partir do predomínio de profissionais do sexo feminino na enfermagem, onde além das responsabilidades e atividades repetitivas das quais são submetidas, ressalta-se a dupla ou tripla jornada ${ }^{7}$. Há ainda a questão biológica de que algumas doenças osteomusculares, como a síndrome de túnel do carpo, um dos tipos de LER/ DORT, uma neuropatia compressiva, é mais comum em mulheres do que em homens ${ }^{20}$.

Além das questões específicas, as mudanças tecnológicas, a crise financeira e econômica, a escassez de recursos físicos, materiais e humanos que sobrevieram sobre as organizações, incluindo as de saúde, demandam maiores atribuições aos enfermeiros, fazendo com que esta categoria sofra com as inadequadas condições de trabalho, 0 acúmulo de tarefas e com a insalubridade do ambiente 4 .

Destaca-se que além dos regimes de trabalho diários $31,3 \%$ dos entrevistados possuem 30 horas adicionais em outras unidades de saúde, demonstrando que estão sujeitos à sobrecarga profissional ${ }^{17}$.

Considerando que entre os principais fatores de risco relacionados aos distúrbios musculoesqueléticos estão: a organização do trabalho (aumento da jornada de trabalho, horas extras excessivas, ritmo acelerado, déficit de trabalhadores); os fatores ambientais (mobiliários inadequados, iluminação insuficiente) e as possíveis sobrecargas de segmentos corporais em determinados movimentos, por exemplo: força excessiva para realizar determinadas tarefas, repetitividade de movimentos e de posturas inadequadas no desenvolvimento das atividades laborais ${ }^{19}$. Essa sobrecarga as predispõem a um maior adoecimento e afastamentos dos profissionais de enfermagem por lesões físicas, sejam elas reversíveis ou não, incapacidades permanentes ou parciais ${ }^{21}$.

Os trabalhadores que atuam em hospitais estão vulneráveis a sofrer acidentes de trabalho e doenças do trabalho, gerando gasto ${ }^{14}$. Apesar da grande quantidade de trabalhadores acometidos pela DORT é difícil estabelecer estatísticas fidedignas, pois até mesmo os levantamentos oficiais do governo não conseguem retratar a real situação de adoecimento no Brasil ${ }^{21}$, visto o fenômeno da subnotificação.

Verificando-se a faixa etária e o tempo de formado constata-se pouca experiência no trabalho, tratar-se de um grupo relativamente jovem, pois 10 (40\%) têm de 27 a 35 anos, e 11 (44\%) têm de um a três anos de serviço, tornando-se fundamental realizar a prevenção/redução antes da instalação das doenças ${ }^{22}$.

Do total, 23 (92\%) trabalham em regime de 12 por 36 horas. Este regime é relativamente comum dentro das estruturas organizacionais de trabalho nas instituições hospitalares, permitindo a esses profissionais mais de um vínculo empregatício ${ }^{23}$. Dentro deste grupo profissional, as longas jornadas podem ocasionar exaustão, fadiga e até mesmo afetar a assistência aos pacientes, além de interferir na qualidade de vida dos trabalhadores ${ }^{24}$. Um estudo iraniano demonstrou que sintomas musculoesqueléticos aumentam em enfermeiros que trabalhavam em horário estendido e com postura de trabalho instável e que esses sintomas estão associados direta e indiretamente às condições de trabalho físico e mental, à situação profissional, à idade e ao estilo de vida ${ }^{25}$.

Esse ritmo acelerado da enfermagem interfere diretamente no processo saúde-doença dos profissionais, desencadeando alterações psíquica/física, podendo ser irritabilidade, ansiedade, tristeza, insônia, frustração, hipertensão, taquicardia e modificação do fluxo menstrual, decorrentes do ritmo de trabalho ${ }^{1}$, fato identificado na amostra em que $7(28 \%)$ informaram que exercem suas atividades laborais em até duas outras instituições. É visível que o exercício profissional em mais de uma instituição representa desgaste físico e mental que, cedo ou tarde, pode repercutir em agravos a saúde ${ }^{26}$.

Em um estudo realizado com profissionais de enfermagem em Salvador constatou-se que, além da dor lombar, teve destaque as dores no ombro, pescoço e parte alta do dorso ${ }^{19}$. No presente estudo, os braços aparecem como a localização mais frequente de dor, interferindo na realização de todas as atividades.

Os afastamentos dos trabalhadores de enfermagem muitas vezes são desencadeados pela dor ou desconforto musculoesquelético. Em um estudo constatou-se que $96,3 \%$ dos profissionais de enfermagem relataram dor nos últimos 12 meses e 73,1\% nos últimos sete dias, o que demonstra íntima ligação da DORT com o afastamento dos trabalhadores ${ }^{27}$.

As incapacidades parciais ou totais podem ocasionar o absenteísmo dos profissionais de enfermagem, o que torna um fator limitante para a organização hospitalar, problema relacionado a questões estruturais, organizacionais e condições laborais em que esse trabalhador é exposto ${ }^{14}$. A licença médica norteia as decisões gerencias e organizacionais 
do hospital. A categoria de técnicos e auxiliares de enfermagem alcançou um total de $84 \%$ de licença médica e os dias de afastamento foram de 3 a 15 dias totalizando 1.319 dias anuais $(41 \%)^{28}$.

Considerando que a LER/DORT não é simplesmente uma lesão causada por um esforço repetitivo qualquer e que sua complexidade está além dos sintomas físicos, incluindo-se a organização do trabalho, as dificuldades interpessoais bem como os fatores ergonômicos ${ }^{26}$, o Ministério da Saúde estabelece diretrizes sobre prevenção, diagnóstico, tratamento e reabilitação, com o intuito de auxiliar os profissionais, para que sinais e sintomas sejam identificados precocemente, evitando incapacidades e dependências pós evento ${ }^{29}$. No entanto a saúde do trabalhador, em especial da área de enfermagem, apesar de seus avanços significativos ainda há falhas nas estratégias de promoção e prevenção dos DORT entre trabalhadores de enfermagem ${ }^{27}$.

Para implantar essas estratégias, é preciso um novo olhar para esta questão, incluindo aspectos da condição de vida, trabalho e saúde dos trabalhadores e uma intervenção humanizada, integral e imparcial. Além disso, é fundamental a reorganização do processo de trabalho, da implementação de políticas públicas e visão ampliada do processo saúde doença, objetivando redução dos custos financeiros quanto a afastamentos, indenizações e principalmente o sofrimento dos trabalhadores expostos².

Também é necessário observar as especificidades de cada trabalhador perante o enfrentamento do processo diagnóstico, tratamento e reabilitação, articulando ações individuais e coletivas, institucionais e interinstitucionais, setoriais e transetoriais para que haja uma efetiva reabilitação destes profissionais no seu cotidiano de trabalho ${ }^{10}$. Além da prevenção, a reabilitação do profissional de saúde é importante políticas de reintegração dos trabalhadores com incapacidade ${ }^{4,28}$, visto que a sensação de dependências e a depressão são fenômenos constantes nos trabalhadores com incapacidades ${ }^{2}$.

O papel do enfermeiro em relação a LER/DORT, está diretamente ligado à promoção e prevenção da saúde, seja no planejamento, execução e/ou avaliação da educação em saúde, efetuando ações capazes de criar ambientes ergonomicamente adequados e saudáveis ${ }^{27}$. 0 desafio da enfermagem é gerenciar a efetividade das ações, 0 uso de tecnologias para o desenvolvimento de trabalhos, as pausas durante a jornada, a administração dos conflitos e a melhoria do clima organizacional ${ }^{14}$ para que as ações educativas e assistenciais no âmbito da saúde ocupacional tornando efetivas a melhora na qualidade de vida dos trabalhadores ${ }^{30}$.

Como qualquer pesquisa, o presente estudo apresenta limitações pelo próprio método adotado que não incluiu observação da realização das atividades realizadas pelos profissionais de enfermagem a fim de compreender a dinâmica corporal e a adoção ou não de medidas preventivas. Também não foram avaliadas demandas físicas e psicossociais no trabalho. Recomenda-se outros estudos que abordem a obsrevação dos postos de trabalho, bem como avaliação das demandas físicas e psicossociais no trabalho.

\section{Considerações Finais}

No presente estudo, a maioria é do sexo feminino, com idades de 28 a 37 anos, com mais de três anos de formação, trabalham em regime de turno. Além disso, boa parte dos participantes têm outros vínculos empregatícios, conseguem identificar sinais e sintomas das LER/DORT, apesar de não procurarem tratamento médico. A dor foi elencada pela maioria dos sujeitos e relacionada às atividades de grande esforço especialmente as de transferência do paciente.

Os resultados sugerem como medidas preventivas estratégias de estrutura ergonômica, aliada a educação continuada, ginástica laboral, planejamento das atividades, equipamentos que auxiliem a equipe de enfermagem no manuseio com o paciente e reorganização do ambiente de trabalho.

O enfermeiro tem um papel importante na educação e na implantação da ergonomia, conciliando uma melhora e adequação do posto de trabalho e trabalhando junto aos seus colaboradores uma nova cultura de hábitos saudáveis capazes de promover um conhecimento e conscientização de prevenção e promoção da saúde para o desenvolvimento das atividades laborativas. 


\section{Referências}

1. Mauro MYC, Paz AF da, Mauro CCC, Pinheiro MA de S, Silva VG. Working conditions of the nursing team in the patient wards of a university hospital. Esc Anna Nery. junho de 2010;14(2):244-52.

2. Caetano VC, Cruz DT, Silva GA, Leite ICG, Carvalho SM. Health-disease process: a study of social representations of workers with WMSDSs. Physis Rev Saúde Coletiva. 2012;22(3):1047-62.

3. Junkes MB, Pessoa VF. Financial expense incurred by medical leaves of health professionals in Rondonia public hospitals, Brazil. Rev Lat Am Enfermagem. 2010;18(3):406-12.

4. Saldanha JHS, Pereira APM, Neves R da F, Lima MAG de. Facilitating factors and barriers for returning to work in workers affected by Repetitive Strain Injury (RSI)/Work-Related Musculoskeletal Disorders (WMSDs). Rev Bras Saúde Ocupacional. junho de 2013;38(127):122-38.

5. Munabi IG, Buwembo W, Kitara DL, Ochieng J, Nabirye RC, Mwaka ES. Musculoskeletal disorders among nursing staff: a comparison of five hospitals in Uganda. Pan Afr Med J [Internet]. 2014 [citado 10 de setembro de 2014];17. Recuperado de: http://www.panafrican-med-journal.com/content/article/17/81/full/

6. Ribeiro NF, Fernandes R de CP, Solla DJF, Junior S, Costa A, Junior S, et al. Prevalence of musculoskeletal disorders in nursing professionals. Rev Bras Epidemiol. junho de 2012;15(2):429-38.

7. Duarte AF, de Souza APC, Macedo A de F, de Araujo FF, Passos JP, Pereira C de A. Fatores de riscos para distúrbios osteomusculares relacionados ao trabalho-DORT em profissionais de enfermagem. Rev Pesqui Cuid É Fundam Online. 2012;53-6. 8. Moraes PWT, Bastos AVB. As LER/DORT e os fatores psicossociais. Arq Bras Psicol. junho de 2013;65(1):02-20.

9. Scopel J, Oliveira PAB, Wehrmeister FC. RSI/WRMSD in the third decade after restructuring of banking: new associated factors? Rev Saúde Pública. outubro de 2012;46(5):875-85.

10. Brasil. Dor relacionada ao trabalho : lesões por esforços repetitivos (LER) : distúrbios osteomusculares relacionados ao trabalho (DORT) [Internet]. Editora do Ministério da Saúde; 2012 [citado 17 de setembro de 2014]. Recuperado de: http://www.cvs.saude.sp.gov.br/up/DORT\%20LER\%20(MS.MMaeno\%20et\%20alii)\%202012.pdf

11. Smith DR. Musculoskeletal complaints and psychosocial risk factors among Chinese hospital nurses. Occup Med. 1 de dezembro de 2004;54(8):579-82.

12. Smith DR, Mihashi M, Adachi Y, Koga H, Ishitake T. A detailed analysis of musculoskeletal disorder risk factors among Japanese nurses. J Safety Res. 2006;37(2):195-200.

13. Fonseca R, Serranheira F. Sintomatologia músculo-esquelética auto-referida por enfermeiros em meio hospitalar. Rev Port Saude Publica. 2006;6:37-44.

14. Lelis CM, Battaus MRB, Freitas FCT de, Rocha FLR, Marziale MHP, Robazzi ML do CC. Work-related musculoskeletal disorders in nursing professionals: an integrative literature review. Acta Paul Enferm. 2012;25(3):477-82.

15. Fernandes R de CP. Distúrbios musculoesqueléticos e trabalho industrial [Tese de Doutorado]. [Salvador]: Instituto de Saúde Coletiva da Universidade Federal da Bahia; 2004.

16. Brasil. Resolução CNS no. 466, de 12 de dezembro de 2012-Estabelece as diretrizes e normas brasileiras regulamentadoras de pesquisas envolvendo seres humanos [Internet]. 2012. Recuperado de: http://www.rbbioetica.com. br/submissao/index.php/RBB/article/view/79

17. Beleza F, Maria C, Teles de Oliveira Gouveia M, Robazzi DCC, Lúcia M, Torres D, et al. Riesgos ocupacionales y problemas de salud percibidos por trabajadores de enfermería en una unidad hospitalaria. Cienc Enferm. 2013;19(3):63-71. 18. Costa FM, Vieria MA, Sena $\mathrm{R}$ de. Absenteísmo relacionado à doenças entre membros da equipe de enfermagem de um hospital escola os da equipe de enfermagem de um hospital escola. Rev Bras Enferm. 2009;62(1):38-44.

19. Ribeiro NF, Fernandes R de CP, Solla DJF, Junior S, Costa A, Junior S, et al. Prevalence of musculoskeletal disorders in nursing professionals. Rev Bras Epidemiol. junho de 2012;15(2):429-38.

20. Carvalho KMD de, Soriano EP, Carvalho MVD de, Mendoza CC de, Vidal HG, Araújo ABVL. Level of evidence and grade of recommendation of articles on the diagnostic accuracy of ultrasonography in carpal tunnel syndrome. Radiol Bras. abril de 2011;44(2):85-9.

21. Moreira RFC, Sato TO, Foltran FA, Silva LCCB, Coury HJCG. Prevalence of musculoskeletal symptoms in hospital nurse technicians and licensed practical nurses: associations with demographic factors. Braz J Phys Ther. agosto de 2014;18(4):323-33. 
22. De D, Wynn E. Preventing muscular contractures through routine stroke patient care. Br J Nurs Mark Allen Publ. 24 de agosto de 2014;23(14):781-6.

23. Vendemiatti M, Siqueira ES, Filardi F, Binotto E, Simioni FJ. Hospital management conflict: the leadership role. Ciênc Amp Saúde Coletiva. junho de 2010;15:1301-14.

24. Silva AA, Rotenberg L, Fischer FM. Nursing work hours: individual needs versus working conditions. Rev Saúde Pública. dezembro de 2011;45(6):1117-26.

25. Dehdashti A. A structural equation modelling method to describe work environment risk factors and musculoskeletal symptoms among hospital nurses. Occup Environ Med. 1 de junho de 2014;71(Suppl_1):A68-A68.

26. Barbosa M do SA, Santos RM dos, Trezza MCSF. Worker's life before and after Repetive Cumulative Trauma (RCT) and Osteomuscular Work-Related Disease (OWRD). Rev Bras Enferm. outubro de 2007;60(5):491-6.

27. Magnago TSB de S, Lisboa MTL, Griep RH, Kirchhof ALC, Camponogara S, Nonnenmacher C de Q, et al. Nursing workers: work conditions, social-demographic characteristics and skeletal muscle disturbances. Acta Paul Enferm. 2010;23(2):187-93.

28. Estorce TP, Kurcgant P. Sick leave and nursing personnel management. Rev Esc Enferm USP. outubro de 2011;45(5):1199-205.

29. Grabara M, Nawrocka A, Niestrój-Jaworska M, Wołkowycka B, Cholewa J. [Physical recreational activity and musculoskeletal disorders in nurses]. Med Pr. 2014;65(2):181-8.

30. Mata M de S, Costa FA da, Souza TO de, Mata ÁN de S, Pontes JF. Pain and functionality in primary health care. Ciênc Amp Saúde Coletiva. janeiro de 2011;16(1):221-30.

\section{Cristiane Aparecida Silveira}

Endereço para correspondência - Rua: Pe. Francis Cletus Cox, n 1661, Bairro: Jardim Country Club, CEP: 37701355, Poços de Caldas, MG, Brasil.

E-mail: casilve@yahoo.com.br

Lattes: http://lattes.cnpq.br/8840798305145545

Camila Gonçalves Monteiro Anunciação - camonteiro01@yahoo.com.br

Luciana Aparecida Sales - jbsales@pocos-net.com.br

Mileid Correa de Andrade - mileidenf@hotmail.com

Sônia Maria Alves de Paiva - paiva@pucpcaldas.br

\section{Enviado em 10 de dezembro de 2015. Aceito em 18 de maio de 2016.}


